\title{
The Loss-Averse Newsvendor Problem with Random Capacity
}

\author{
Chongfeng Lan ${ }^{1,2}$, Huanyong $\mathrm{Ji}^{1}$ and Jing $\mathrm{Li}^{3}$ \\ ${ }^{1}$ School of Economics and Management, Beijing University of Posts and \\ Telecommunications, \\ ${ }^{2}$ School of Economics, Fuyang Normal College, \\ ${ }^{3}$ School of economic and management, Tian Jin University of Technology and \\ Education (China) \\ ${ }^{1}$ lchfym@sina.com; ${ }^{2}$ jihuanyong1986@126.com; ${ }^{3}$ smartlij@163.com
}

\begin{abstract}
This paper examines a single-period inventory problem with random capacity and demand, where the loss-averse preference is adopted to describe the newsvendor's decision-making behavior. When the loss-averse newsvendor orders, the supplier has some random replenishment capacity. He will choose an order quantity to maximize his expected utility. The newsvendor's optimal ordering policy is obtained, then the impacts of loss aversion, price, salvage value and cost on the optimal order quantity are analysed. Our research shows that the loss-averse newsvendor's optimal order quantity is always less than the risk-neutral newsvendor's. Moreover, the newsvendor's optimal order quantity and expected utility are decreasing in the loss aversion level and purchasing cost, while increasing in selling price and salvage value. The numerical experiments are conducted to demonstrate our theoretical results.
\end{abstract}

Keywords: inventory; newsvendor problem; loss aversion; random capacity

\section{Introduction}

The newsvendor problem is the foundation of many stochastic inventory control models, which has been studied for a long time and applied in a broad array of business settings. The standard newsvendor model is based upon risk neutrality so that managers will select an order quantity to maximize expected profit or minimize expected cost. However, experimental evidence (Fisher and Raman, 1996; Kahn, 1992; Feng et. al., 2011) suggests that the newsvendor's decisions often challenge the risk-neutral assumption. The assumption of risk-neutrality therefore seems to be inadequate for contemporary supply chain management. In view of this, a number of papers have been devoted to risk analysis of supply chain models. For an extensive review of the literature on supply chain risk management or extensions of different objectives for the newsvendor problem, the reader is referred to Khouja (1999), Tang (2006) and Qin et. al., (2011).

We refer to the deviation of the newsvendor's optimal order quantity from the profit maximization order quantity as decision bias in the newsvendor problem (Wang and Webster, 2009). One major reason why decision bias may exist in the newsvendor problem is that a manager may have loss aversion rather than risk neutrality. Loss aversion, which is one of the key features of the Prospect Theory (Kahneman and Tversky, 1979), means that people are more sensitive to losses than to same-sized gains, and the perception of gains or losses is related to the reference point. Since loss aversion is intuitively appealing and supported in economics and management, etc., incorporating it into the inventory model has become an important and growing area of research.

Recently, the inventory model based on loss aversion has been studied in many works. As far as we know, Schweitzer and Cachon (2000) study in the first place a loss-averse newsvendor issues. They derive that a loss-averse newsvendor without shortage cost will 
order strictly less than a risk-neutral newsvendor, and the optimal order quantity decreases in the degree of loss aversion. Wang and Webster (2009) further consider the shortage cost and find that the impact of loss aversion on the optimal order quantity to be different from that find in Schweitzer and Cachon (2000). Wang (2010) investigates the loss-averse newsvendor game in which the total demand is allocated proportionally to the newsvendor's order quantities. There exists a unique Nash equilibrium in this game. Liu et. al., (2013) study a loss-averse newsvendor problem with random yield and demand. They consider both shortage cost and no shortage cost, respectively. For the model without shortage cost, the loss-averse retailer's optimal order quantity is always less than the risk-neutral retailer's, and decreasing in the loss aversion level. While for the model with shortage cost, the loss-averse retailer's optimal order quantity may be larger than the risk-neutral retailer's, and increasing in the loss aversion level. Chen et. al., (2014) investigate a one-period two-echelon supply chain composed of a risk-neutral supplier that produces short life-cycle products and a loss-averse retailer that orders from the supplier via option contracts and sells to end-users with stochastic demand in the selling season. They find that the loss-averse retailer may order less than, equal to, or more than the risk-neutral retailer. Further, they show that the loss-averse retailer's optimal order quantity may increase in retail price and decrease in option price and exercise price, which is different from the case of a risk-neutral retailer. Ma et. al., (2012) discuss loss-averse newsvendor model with two ordering opportunities and market information updating. They derive the retailer's optimal ordering decisions and analyzed the monotonicity behaviours of the critical market signal, the optimal first-stage order quantity, and the optimal expected payoff with respect to the penalty coefficient. Moreover, they examine the impact of demand forecast quality on the retailer's decisions. Other papers considering the loss-averse preferences include Shen et. al., (2011), Deng et. al., (2013), Wang and Webster (2007) and Lee et. al., (2015), among others.

The major source of randomness in inventory models is the demand. However, the uncertainty of demand is not necessarily the only source of randomness. In fact, in recent years, there has been a lot of emphasis on models with supply uncertainty as well. These include yield loss (related to quality considerations), unreliable machinery and unplanned maintenance. We refer interest readers to Yano and Lee (1995) and Grosfeld-Nirand and Gerchak (2004) for summaries of the effects of random capacity on inventory control.

Random capacity, resulting from the before mentioned uncertainties, has been studied for the well-known newsvendor problem. Ciarallo et. al., (1994) first study of the implications of variable capacity for the newsvendor problem. They study a periodic-review production model with random capacity, and show for the single-period problem that random capacity does not affect the optimal order quantity. Wang and Gerchak (1996) extend their model to a system with both random capacity and random yield. Erdem et. al., (2006) consider an EOQ model with multiple suppliers who face random capacities. They obtain the unique optimal order quantities for the suppliers under which the expected unsatisfied order is the same for each supplier, and study the diversification among suppliers for some given distributions. Wu et. al., (2013) study the effect of capacity uncertainty on the inventory decisions of a risk-averse newsvendor with two well-known risk criteria, namely VaR included as a constraint and CVaR. They find that capacity uncertainty decreases the order quantity under the CVaR criterion. While under the VaR constraint, capacity uncertainty leads to an order decrease for low confidence levels, but to an order increase for high confidence levels. Okyay et. al., (2014) consider the classical newsvendor problem with random demand, random yield and/or random capacity where the demand and supply are assumed to be dependent. They provide mathematical characterizations of the optimal order quantity.

A common assumption of all the literature on loss aversion is, to the best of our knowledge, that it has not been studied on inventory systems with random capacity. An interesting and unexplored question is what happens in an, arguably even more realistic, 
situation with random capacity. This is the main focus of our research. Motivated by Schweitzer and Cachon (2000), Wang and Webster (2009) and Wu et. al., (2013), we investigate a single-period inventory problem in which random capacity and loss aversion are jointly considered. The combined randomness of demand and capacity enhances the level of uncertainty, thus leading to an increased complexity of the model. The loss-averse newsvendor's objective is to maximize his expected utility without shortage cost. We focus on the following questions: (1) How will the loss-averse newsvendor determine his optimal order quantity when capacity is random? (2) What is the impact of loss aversion on the optimal order quantity? (3) How will the optimal order quantity change as price, salvage value or cost increases? More specifically, we find that the loss-averse newsvendor's optimal order quantity is always less than the risk-neutral newsvendor's. Moreover, the newsvendor's optimal order quantity and expected utility are decreasing in the loss aversion level and purchasing cost, while increasing in selling price and salvage value.

The rest of this paper is organized as follows. In Section 2, we formulate the loss-averse newsvendor model with random capacity and demand. We derive the optimal order quantity and investigate comparative statics on the loss-averse newsvendor's optimal order quantity and expected utility. In Section 3, numerical experiments are presented. Finally, conclusions and suggestions for future research are given in Section 4.

\section{The Loss-Averse Newsvendor Model with Random Capacity}

Consider a newsvendor problem with random demand and capacity. We adopt the commonly used random capacity model that is mentioned by Wu et. al., (2013). Random demand and random capacity are assumed to be independent. Without loss of generality, we assume that $F(0)=G(0)=0$. Notations concerned in this paper are listed as follows:

- c: purchasing cost per unit.

- $\quad$ : selling price per unit, $c<p$.

- $\quad$ s: salvage value per unit, $s<c$.

- q: order quantity.

- $\quad \mathrm{Q}(\mathrm{q})$ : amount of newsvendor received.

- $\mathrm{X}$ : random demand. Its probability density function is $\mathrm{f}(\mathrm{x})$ and cumulative distribution function is $\mathrm{F}(\mathrm{x})$.

- Y: random capacity, i.e., the supplier has some random replenishment capacity so that $\mathrm{Q}(\mathrm{q})=\min \{\mathrm{Y}, \mathrm{q}\}$. Its probability density function is $\mathrm{g}(\mathrm{y})$ and cumulative distribution function is $\mathrm{G}(\mathrm{y})$.

- $q_{\lambda}^{*}$ : newsvendor's optimal order quantity.

- $\quad E_{\lambda}^{*}$ : newsvendor's optimal expected utility.

For any given realized demand $\mathrm{x}$ and capacity $\mathrm{y}$, the newsvendor's profit is

$$
\begin{aligned}
\pi(q, x, y)= & (p-c) \min \{q, y\}-(p-s)(\min \{q, y\}-x)^{+} \\
& =\left\{\begin{array}{l}
(s-c) y+(p-s) x \quad x<y \leq q, \\
(p-c) y \quad y \leq q, y \leq x, \\
(s-c) q+(p-s) x \quad x<q<y, \\
(p-c) q \quad q<y, q \leq x .
\end{array}\right.
\end{aligned}
$$

Without loss of generality, we assume that the newsvendor's initial wealth (reference level) is 0 at the beginning of the selling season, and we take into consideration a simple piecewise linear form of newsvendor's loss aversion utility function 
$U(\pi)= \begin{cases}\pi, & \pi \geq 0 \\ \lambda \pi, & \pi<0\end{cases}$

where $\lambda$ is the newsvendor's loss aversion coefficient. We assume $\lambda \geq 1$, which means that people are more sensitive losses than to same-sized gains. Higher value of $\lambda$ corresponds to a higher level of newsvendor's loss aversion. This piecewise-linear form of loss aversion utility function has been widely used in the economics, finance, and operations management literature (Schweitzer and Cachon, 2000; Barberis and Huang, 2001; Wang and Webster, 2009), and is an approximation of the non-linear (and hence leading to intractability of the model) utility functions.

After mapping the newsvendor's profit function (1) into his utility function (2), we can express the newsvendor's expected utility $E[U(\pi(q, x, y))]$ as

$$
\begin{aligned}
& E[U(\pi(q, x, y))] \\
& =\lambda \int_{0}^{q} \int_{0}^{\frac{(c-s) y}{p-s}}[(s-c) y+(p-s) x] d F(x) d G(y)+\int_{0}^{q} \int_{\frac{(c-s) y}{p-s}}^{y}[(s-c) y+(p-s) x] d F(x) d G(y) \\
& +\lambda \int_{q}^{\infty} \int_{0}^{\frac{(c-s) q}{p-s}}[(s-c) q+(p-s) x] d F(x) d G(y)+\int_{q}^{\infty} \frac{\int_{(c-s) q}^{q}}{p-s}[(s-c) q+(p-s) x] d F(x) d G(y) \\
& +\int_{0}^{q} \int_{y}^{\infty}(p-c) y d F(x) d G(y)+\int_{q}^{\infty} \int_{q}^{\infty}(p-c) q d F(x) d G(y) \\
& =E[\pi(q, x, y)]+ \\
& (\lambda-1)\left\{\int_{0}^{q} \int_{0}^{\frac{(c-s) y}{p-s}}[(s-c) y+(p-s) x] d F(x) d G(y)+\int_{q}^{\infty} \int_{0}^{\frac{(c-s) q}{p-s}}[(s-c) q+(p-s) x] d F(x) d G(y)\right\} .
\end{aligned}
$$

The expected utility of loss-averse newsvendor with random capacity is the expected profit plus the expected losses, biased by a factor of $\lambda-1$. If $\lambda=1$, then the newsvendor is risk neutral and the second term in (3) drops out.

Theorem 1. $E[U(\pi(q, x, y))]$ is concave in $\mathrm{q}$ and there exists a unique optimal order quantity $q_{\lambda}^{*}$ that maximizes the expected utility of loss-averse newsvendor with random capacity and satisfies the following first-order condition:

$(\lambda-1)(c-s) F\left(\frac{(c-s) q_{\lambda}^{*}}{p-s}\right)+(p-s) F\left(q_{\lambda}^{*}\right)=p-c$.

Proof. Taking the first-order derivative of $E[U(\pi(q, x, y))]$ with respect to $q$ and applying the chain rule, we get 


$$
\begin{aligned}
& \frac{d E[U(\pi(q, x, y))]}{d q} \\
& =(\lambda-1)(s-c) q g(q) \int_{0}^{\frac{(c-s) q}{p-s}} d F(x)+(\lambda-1)(s-c) \int_{q}^{\infty} \int_{0}^{\frac{(c-s) q}{p-s}} d F(x) d G(y) \\
& -(\lambda-1)(s-c) q g(q) \int_{0}^{\frac{(c-s) q}{p-s}} d F(x)+(\lambda-1)(s-c) q \frac{(c-s)}{p-s} f\left(\frac{(c-s) q}{p-s}\right)(1-G(q)) \\
& +(\lambda-1)(p-s) g(q) \int_{0}^{\frac{(c-s) q}{p-s}} x d F(x)-(\lambda-1)(p-s) g(q) \int_{0}^{\frac{(c-s) q}{p-s}} x d F(x) \\
& +(\lambda-1)(c-s) \frac{(c-s) q}{p-s} f\left(\frac{(c-s) q}{p-s}\right)(1-G(q))+\frac{d E[\pi(q, x, y)]}{d q} \\
& =(\lambda-1)(s-c) \bar{G}(q) F\left(\frac{(c-s) q}{p-s}\right)+(p-c) \bar{G}(q)-(p-s) \bar{G}(q) F(q) \\
& =\left[(\lambda-1)(s-c) F\left(\frac{(c-s) q}{p-s}\right)+(p-c)-(p-s) F(q)\right] \bar{G}(q)
\end{aligned}
$$

where $\bar{G}(q)=1-G(q)$ denotes the tail distribution. Let

then

$$
\tau(q)=(\lambda-1)(s-c) F\left(\frac{(c-s) q}{p-s}\right)+(p-c)-(p-s) F(q),
$$

$\frac{d \tau(q)}{d q}=(s-p) f(q)-(\lambda-1) \frac{(c-s)^{2}}{p-s} f\left(\frac{c-s}{p-s} q\right)<0$.

Let $q_{\lambda}^{*}$ satisfy $\tau\left(q_{\lambda}^{*}\right)=0$, then by (6), we have

(i) when $q<q_{\lambda}^{*}$, $\frac{d E[U(\pi(q, x, y))]}{d q}=\tau(q) \cdot \bar{G}(q)>0$;

(ii) when $q>q_{\lambda}^{*}$, $\frac{d E[U(\pi(q, x, y))]}{d q}=\tau(q) \cdot \bar{G}(q)<0$.

Thus $E[U(\pi(q, x, y))]$ is concave in q. Let $\frac{d E[U(\pi(q, x, y))]}{d q}=0$, then the unique optimal order quantity $q_{\lambda}^{*}$ of loss-averse newsvendor with random capacity satisfies (4).

If the newsvendor with random capacity is risk-neutral, i.e., $\lambda=1$, then the first-order condition (4) reduces to

$$
(p-s) F\left(q_{1}^{*}\right)=p-c,
$$

$$
\text { and we get } q_{1}^{*}=F^{-1}\left(\frac{p-c}{p-s}\right) \text {, it }
$$

it is obvious that the uncertainty of capacity does not affect the newsvendor's optimal order quantity. Indeed, this result is intuitive, as the newsvendor gains nothing by ordering more if the capacity is uncertain, and receives a lower profit by ordering less.

Theorem 2. For any $\lambda>1, \quad q_{\lambda}^{*}<q_{1}^{*}$. 
Proof. Let $q_{\lambda}^{*}=q_{1}^{*}=F^{-1}\left(\frac{p-c}{p-s}\right)$ and take it into the left side of equation (4), then $(\lambda-1)(c-s) F\left(\frac{(c-s) q_{\lambda}^{*}}{p-s}\right)+(p-s) F\left(q_{\lambda}^{*}\right)>p-c$. Since the left side of equation (4) is increasing in $q_{\lambda}^{*}$. Thus for any $\lambda>1, q_{\lambda}^{*}<q_{1}^{*}$.

Theorem 2 states that if the shortage cost is ignored, then the loss-averse newsvendor with random capacity will order less than the risk-neutral newsvendor. This result is consistent with those of Schweitzer and Cachon (2000).

To gain more insights, we investigate comparative statics of loss aversion level, price, cost and salvage value changes on the loss-averse newsvendor's optimal order quantity and expected utility.

Theorem 3. For any $\lambda>1$, the newsvendor's optimal order quantity and expected utility are decreasing in $\lambda$. Moreover, they are decreasing in $c$, while increasing in $p$ and $s$.

Proof. Theorem 3 can be proved taking the first partial derivatives of (4) with respect to each parameter and determining their signs.

Let

$$
H(\lambda, p, c, s)=(\lambda-1)(c-s) F\left(\frac{(c-s) q_{\lambda}^{*}}{p-s}\right)+(p-s) F\left(q_{\lambda}^{*}\right)-(p-c)=0,
$$

then we easily get

$$
\begin{aligned}
& \frac{\partial H(\lambda, p, c, s)}{\partial \lambda}=(c-s) F\left(\frac{(c-s) q_{\lambda}^{*}}{p-s}\right)>0, \\
& \frac{\partial H\left(\lambda, p, c, s_{1}\right)}{\partial q_{\lambda}^{*}}=(\lambda-1) \frac{(c-s)^{2}}{p-s} f\left(\frac{(c-s) q_{\lambda}^{*}}{p-s}\right)+(p-s) f\left(q_{\lambda}^{*}\right)>0 .
\end{aligned}
$$

By the Implicit Function Theorem, from (9) and (10), we have

$$
\frac{\partial q_{\lambda}^{*}}{\partial \lambda}=-\frac{\partial H(\lambda, p, c, s)}{\partial \lambda} / \frac{\partial H\left(\lambda, p, c, s_{1}\right)}{\partial q_{\lambda}^{*}}<0 .
$$

Thus the newsvendor's optimal order quantity is decreasing in $\lambda$.

When $\lambda=\lambda_{1}$, the newsvendor's optimal order quantity is $q_{\lambda_{1}}^{*}$. When $\lambda=\lambda_{2}$, the newsvendor's optimal order quantity is $q_{\lambda_{2}}^{*}$. Without loss of generality, we assume that $\lambda_{1}<\lambda_{2}$, then by the optimality of $q_{\lambda_{1}}^{*}$, we get

$$
E_{\lambda_{1}}^{*}\left[U\left(\pi\left(q_{\lambda_{1}}^{*}, x, y\right)\right)\right] \geq E_{\lambda_{1}}\left[U\left(\pi\left(q_{\lambda_{2}}^{*}, x, y\right)\right)\right] .
$$

Moreover, since expected utility is decreasing in $\lambda$, thus

$$
E_{\lambda_{1}}\left[U\left(\pi\left(q_{\lambda_{2}}^{*}, x, y\right)\right)\right] \geq E_{\lambda_{2}}^{*}\left[U\left(\pi\left(q_{\lambda_{2}}^{*}, x, y\right)\right)\right] \text {. }
$$

Therefore

$$
E_{\lambda_{1}}^{*}\left[U\left(\pi\left(q_{\lambda_{1}}^{*}, x, y\right)\right)\right] \geq E_{\lambda_{2}}^{*}\left[U\left(\pi\left(q_{\lambda_{2}}^{*}, x, y\right)\right)\right]
$$

which implies that the newsvendor's optimal expected utility is decreasing in $\lambda$.

The other results can be proved in a similar way. 
Theorem 3 shows that the loss-averse retailer will order less than the risk-neutral one $(\lambda=1)$, and the more loss-averse the retailer, the less his order quantity. Moreover, the impacts of price, salvage value and cost on the optimal order quantity are consistent with our common sense. These results also hold in the risk-neutral newsvendor model. Thus this paper extends the application of classic newsvendor problem.

\section{Numerical Experiments}

In this section, we perform the numerical experiments to illustrate our results. The parameters are set as follows: $c=10, p=20, s=5$ and $\lambda=2$. Suppose that $X$ is uniformly distributed between 0 and 200, then $F(x)=1 / 200$. From Eq. (4), we can calculate the optimal order quantity $q_{\lambda}^{*}=120$. If the newsvendor with random capacity is risk-neutral, i.e., $\lambda=1$, then the optimal order quantity $q_{1}^{*}=133$. Thus Theorem 2 holds.

In order to investigate the impacts of loss aversion level on the optimal order quantity and expected utility, suppose that $Y$ is uniformly distributed between 100 and 300. Using the above dates, we only need the loss aversion coefficient $\lambda$ changes. Results are presented in Figure 1 and 2.

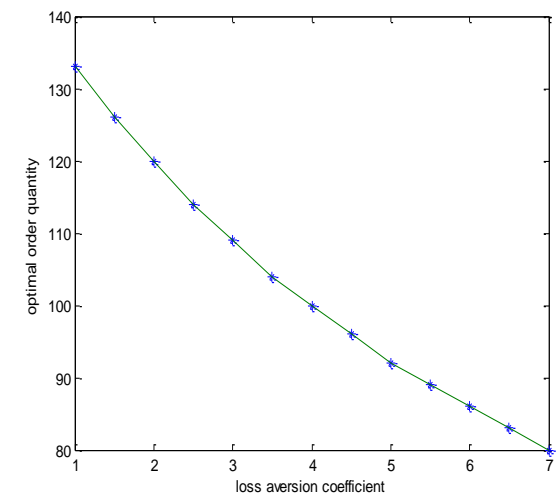

Figure 1. The Impact of Loss Aversion Coefficient $\lambda$ on the Optimal Order Quantity $Q_{\lambda}^{*}$.

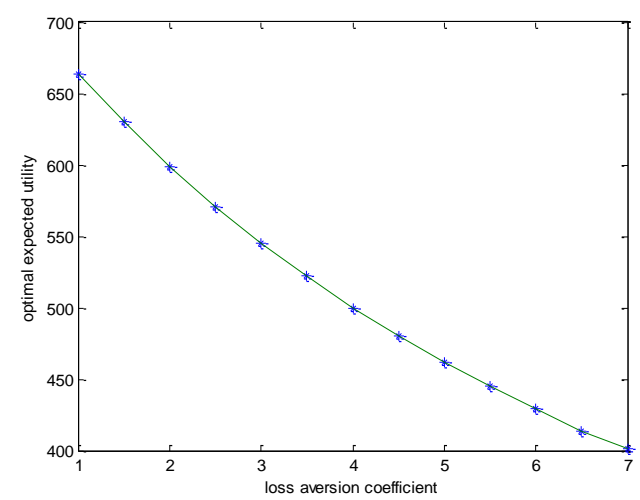

Figure 2. The Impact of Loss Aversion Coefficient $\lambda$ on the Optimal Expected Utility $E_{\lambda}^{*}$.

Figures 1 , and 2, demonstrate that the optimal order quantity $Q_{\lambda}^{*}$ and the optimal expected utility $E_{\lambda}^{*}$ are decreasing in loss aversion level. Therefore, when the loss aversion coefficient $\lambda$ increases, the decision-maker should decrease the order quantity $Q_{\lambda}^{*}$ in order to provide protection against losses and the optimal expected utility $E_{\lambda}^{*}$ correspondingly reduces.

\section{Conclusion}

In this paper, we investigate a loss-averse newsvendor problem with random capacity where the newsvendor's objective is to maximize his expected utility. We use a simple piecewise-linear loss aversion utility function to study the single-period newsvendor model without shortage cost and show that the maximum expected utility is concave in order quantity. Our research shows that the loss-averse newsvendor's optimal order quantity is always less than the risk-neutral newsvendor's. In addition, the newsvendor's optimal order quantity and expected utility are decreasing in the loss aversion level and purchasing cost, while increasing in selling price and salvage value. These results are consistent with our common sense. Our results are illustrated through numerical experiments. 
As a natural extension of our work, future research will consider using a more general utility function to investigate the model with random capacity. Furthermore, the factor of shortage cost can be added in the model. There may be more interesting when you consider the shortage cost, such as Wang and Webster (2009). Finally, the optimal ordering policy for a multi-period problem deserves further study.

\section{Conflict of Interests}

The authors declare that there is no conflict of interests regarding the publication of this paper.

\section{Acknowledgments}

This work was supported by the National Natural Science Foundation of China (71201034) and the Natural Science Research Project of Fuyang Normal College (2014WLGH02).

\section{References}

[1] N. Barberis and M.Huang, "Mental accounting, loss aversion, and individual stock returns", The Journal of Finance, vol. 56, no. 4, (2001), pp. 1247-1292.

[2] X. Chen, G. Hao and L. Li, "Channel coordination with a loss-averse retailer and option contracts", International Journal of Production Economics, vol.150, (2014), pp 52-57.

[3] F. W. Ciarallo, R. Akella and T. E. Morton, "A periodic review, production planning model with uncertain capacity and uncertain demand-optimality of extended myopic policies", Management Science, vol.40, no. 3, (2014), pp. 320-332.

[4] X. Deng, J. Xie and H. Xiong, "Manufacturer-retailer contracting with asymmetric information on retailer's degree of loss aversion", International Journal of Production Economics, vol.142, no. 2, (2013), pp. 372-380.

[5] A. S. Erdem, M. M. Fadiloglu and S. Özekici, "An EOQ model with multiple suppliers and random capacity", Naval Research Logistics, vol. 53, no. 1, (2006), pp. 101-114.

[6] T. Feng, L. R. Keller and X. Zheng, "Decision making in the newsvendor problem: A cross-national laboratory study", Omega, vol. 39, no. 1, (2011), pp. 41-50.

[7] M. Fisher and A. Raman, "Reducing the cost of demand uncertainty through accurate response to early sales", Operations Research, vol. 44, no. 1, (2006), pp. 87-99.

[8] A. Grosfeld-Nir and Y. Gerchak, "Multiple lotsizing in production to order with random yields: Review of recent advances", Annals of Operations Research, vol.126, no. 1-4, (2004), pp. 43-69.

[9] J. A. Kahn, "Why is production more volatile than sales? Theory and evidence on the stockout-avoidance motive for inventory holding", Quarterly Journal of Economics, vol.107, no. 2, (1992), pp. 481-510.

[10] D. Kahneman and A. Tversky, "Prospect theory: An analysis of decision under risk. Econometrica: Journal of the Econometric Society", vol. 47, no. 2, (1979), pp. 263-291.

[11] M. Khouja, "The single-period (news-vendor) problem: literature review and suggestions for future research", Omega, vol. 27, no. 5, (1999), pp. 537-553.

[12] C. Y. Lee, X. Li and M. Yu, "The loss-averse newsvendor problem with supply options", Naval Research Logistics (NRL), vol. 62, no. 1, (2015), pp. 46-59.

[13] W. Liu, S. Song and C. Wu, "The loss-averse newsvendor problem with random yield", Transactions of the Institute of Measurement and Control, (2013).

[14] L, Ma, Y. Zhao, W. Xue, T. C. E. Cheng and H. Yan, "Loss-averse newsvendor model with two ordering opportunities and market information updating", International Journal of Production Economics, vol.140, no. 2, (2012), pp. 912-921.

[15] H. K. Okyay, F. Karaesmen and S. Özekici, "Newsvendor models with dependent random supply and demand", Optimization Letters, vol.8, no. 3, (2014), pp. 983-999.

[16] Y. Qin, R. Wang, A. J. Vakharia, Y. Chen and M. M. Seref, "The newsvendor problem: Review and directions for future research", European Journal of Operational Research, vol. 213, no. 2, (2011), pp:361-374. 
[17] Schweitzer, M. E., \& Cachon, G. P. Decision bias in the newsvendor problem with a known demand distribution: Experimental evidence. Management Science, vol. 46, no. 3, (2000), pp, 404-420.

[18] H. Shen, Z. Pang and T. C. E. Cheng, "The component procurement problem for the loss-averse manufacturer with spot purchase", International Journal of Production Economics, vol. 132, no. 1, (2011), pp. 146-153.

[19] C. S. Tang, "Perspectives in supply chain risk management", International Journal of Production Economics, vol. 103, no. 2, (2006), pp. 451-488.

[20] C. X. Wang, "The loss-averse newsvendor game", International Journal of Production Economics, vol.124, no. 2, (2010), pp. 448-452.

[21] C. X. Wang and S. Webster, "Channel Coordination for a Supply Chain with a Risk-Neutral Manufacturer and a Loss-Averse Retailer”, Decision Sciences, vol. 38, no. 3, (2007), pp. 361-389.

[22] C. X. Wang and S. Webster, "The loss-averse newsvendor problem”, Omega, vol. 37, no. 1, (2009), pp. 93-105.

[23] Y. Wang and Y. Gerchak, "Periodic review production models with variable capacity, random yield, and uncertain demand", Management science, vol. 42, no. 1, (1996), pp. 130-137.

[24] M. Wu, S. X. Zhu and R. H. Teunter, "The risk-averse newsvendor problem with random capacity", European Journal of Operational Research,vol. 231, no. 2, (2013), pp. 328-336.

[25] C. A. Yano and H. L. Lee, "Lot sizing with random yields: A review", Operations Research, vol. 43, no. 2, (1995), pp. 311-334.

\section{Authors}

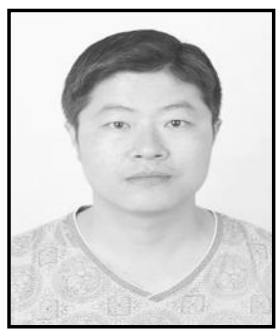

Chongfeng Lan, He received his M.Sc. in Statistics (2010) from Guilin University of Technology. Now he is a lecturer of statistics at School of Economics, Fuyang Normal College. Now he is studying for a doctor's degree in the School of Economics and Management, Beijing University of Posts and Telecommunications, His current research interests include supply chain management and statistics.

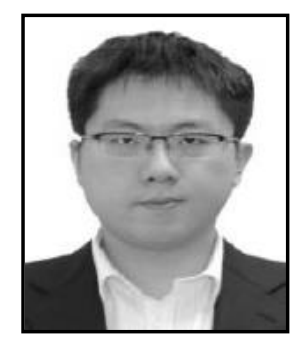

Huanyong Ji, He received his Master Degree in Technology Science (2011) from Hong Kong Baptist University. Now he is studying for a doctor's degree in the School of Economics and Management, Beijing University of Posts and Telecommunications, His current research interests include internet marketing and innovation management.

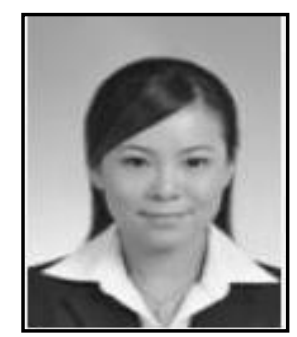

Jing Li, She received her Ph.D in Technical Science (2011) from Beijing University of Posts and Telecommunications. Now she is a full lecturer of electronic commercial Department, Tianjin University of technology and education. Her current research interests include internet marketing and consumer behavior. 
International Journal of $u-$ and e- Service, Science and Technology Vol. 9, No. 12 (2016) 